Quim. Nova, Vol. 36, No. 8, 1232-1235, 2013

\title{
USO DE HARDWARE DE CÓDIGO FONTE ABERTO “ARDUINO” PARA ACIONAMENTO DE DISPOSITIVO SOLENOIDE EM SISTEMAS DE ANÁLISES EM FLUXO
}

\author{
Marcos Y. Kamogawa* \\ Escola Superior de Agricultura "Luiz de Queiroz”, Universidade de São Paulo, Dep. Ciências Exatas, Av. Pádua Dias 11, CP 9, \\ 13418-900 Piracicaba - SP, Brasil \\ Jeová Correia Miranda \\ Centro de Energia Nuclear na Agricultura, Universidade de São Paulo, Av. Centenário 303, CP 96, 13400-970 Piracicaba - SP, \\ Brasil
}

Recebido em 18/12/12; aceito em 27/6/13; publicado na web em 25/7/13

\begin{abstract}
USE OF "ARDUINO" OPEN SOURCE HARDWARE FOR SOLENOID DEVICE ACTUATION IN FLOW ANALYSIS SYSTEMS. This paper describes the use of the open source hardware platform, denominated "Arduino", for controlling solenoid valves for solutions handling in flow analysis systems. System assessment was carried out by spectrophotometric determination of iron (II) in natural water. The sampling rate was estimated as 45 determinations per hour and the coefficient of variation was lower than $3 \%$. Per determination, $208 \mu \mathrm{g}$ of 1-10-phenanthroline and ascorbic acid were consumed, generating $1.3 \mathrm{~mL}$ of waste. "Arduino" proved a reliable microcontroller with low cost and simple interfacing, allowing USB communication for solenoid device switching in flow systems.
\end{abstract}

Keywords: serial port; multicommutation; binary sampling.

\section{INTRODUÇÃO}

O uso de dispositivos eletrônicos, como as válvulas solenoide, válvulas rotatórias e bombas solenoide em sistemas de análises em fluxo, permitiu que as soluções pudessem ser gerenciadas de forma eficiente, controlando os tempos e a sequência de adição. ${ }^{1-3}$ Seu uso possibilita que a estratégia da amostragem binária, ${ }^{4,5}$ utilizada em sistemas com multicomutação, seja realizada com sucesso, melhorando a interação amostra-reagentes e reduzindo o tempo de análise e os problemas de dispersão inerentes aos sistemas de análise em fluxo clássicos. ${ }^{6}$

O acionamento das válvulas ou bombas solenoide em sistemas de análises em fluxo é feita por uma interface eletrônica que compatibiliza o sinal digital enviado pelo computador e a fonte de corrente contínua que alimenta os dispositivos.

Uma das interfaces mais utilizada foi proposta por Torralba et al. ${ }^{7}$ que empregam o circuito integrado ULN2803, que funciona como um interruptor digital, acionado por variações da tensão dos pinos da porta paralela (lpt1) do computador. Todavia, com a evolução dos computadores, as portas paralelas foram substituídas por portas universais como as USB, que padronizou a comunição entre o computador e periféricos e ampliou o número de portas digitais possíveis. Desta forma, as interfaces de controle dos dispositivos solenoide que utilizam a porta paralela ficaram restritas a computadores antigos ou equipamentos encomendados, geralmente de valor superior aos de mercado.

Uma alternativa a esta limitação é o acionamento dos dispositivos solenoide por comunicação serial, entretanto esta necessita de interfaces para conversão do sinal serial, transmitida bit-a-bit. Esta conversão pode ser executada por microcontroladores que podem ser programados para a conversão de sinal ou acionamento de portas digitais.

Microcontroladores do tipo PIC (Programmable Interrupt Controller), são dispositivos de configuração simples, de baixo custo e portáteis, ${ }^{8}$ que permitem a automatização dos sistemas de análises

*e-mail: kamogawa@usp.br sem a necessidade de um microcomputador. Com o dispositivo PIC 16F877 com conversor interno A/D de 10 bits EEPROM é possível transferir e armazenar os resultados analíticos para um computador através de uma saída serial, podendo também este ser lido diretamente na tela de um LCD (Liquid Crystal Display). ${ }^{9}$ Sua eficiência também foi demonstrada em um microssistema de análises por injeção em fluxo com cela de detecção fluorimétrica integrada, que utilizou o microcontrolador PIC 16F819 para automatizar todas as operações do micro-analisador, controlando o acionamento de mini válvulas solenoide e a fotomultiplicadora. ${ }^{10}$

Para usuários leigos em eletrônica a principal dificuldade para o uso dos microcontroladores é a necessidade de construir os circuitos eletrônicos e desenvolver a programação. Neste sentido, o uso da plataforma comercial como o "Arduino" surge como uma alternativa a esta limitação. O Arduino modelo UNO é uma plataforma de hardware de código fonte aberta com comunicação serial, USB, de baixo custo, software livre e de fácil operação. Esta plataforma utiliza microcontroladores da Atmel de 10 bits e componentes complementares que facilitam a programação e a interligação com outros circuitos. O Arduino está disponível comercialmente em diferentes modelos, os quais possuem de 14 a 54 pinos digitais de entrada e saída de dados, que podem ser utilizadas para aquisição de dados ou controlar dispositivos, como bomba peristáltica, válvulas e bombas solenoide.

O objetivo desta nota técnica foi demonstrar o acionamento de válvulas solenoide através da plataforma Arduino, para o gerenciamento de soluções em sistemas de análise em fluxo. A potencialidade do microcontrolador foi comprovada através da determinação de ferro em amostras de água empregando multicomutação em fluxo e amostragem binária.

\section{MATERIAL E MÉTODOS}

\section{Reagentes e soluções}

Todas as soluções foram preparadas com água deionizada e reagentes com grau de pureza analítico. Soluções de referência contendo 
0,5; 1,0; 2,0; 3,0 e 5,0 $\mathrm{mg} \mathrm{L}^{-1} \mathrm{de} \mathrm{Fe}^{3+}$ a partir de $\mathrm{FeCl}_{3}$ (Merck) foram preparadas em meio $0,014 \mathrm{~mol} \mathrm{~L}^{-1}$ de $\mathrm{HNO}_{3}$ (Merck). Como reagente cromogênico foi utilizada solução $0,25 \%(\mathrm{~m} / \mathrm{v})$ de 1,10 -fenantrolina (Merck). Para redução de ferro (III) foi empregada solução 0,5\% $(\mathrm{m} / \mathrm{v})$ de ácido ascórbico (Merck). Solução 0,014 mol L-1 de $\mathrm{HNO}_{3}$ foi utilizada como solução transportadora.

\section{Módulo de análise}

O módulo de análises foi construído utilizando quatro válvulas solenoide de três vias, Figura 1. Para as linhas de transmissão e reatores helicoidais (Figura 1, B) utilizaram-se tubos de polietileno, com diâmetro interno de $0,8 \mathrm{~mm}$. Para propulsão dos fluidos foi utilizada uma bomba peristáltica com 8 canais (Ismatec, IPC-8) e tubos Tygon ${ }^{\circledR}$ com 1,4 mm de diâmetro interno. Para a introdução dos reagentes foi utilizado um conector de acrílico, com cinco canais direcionados a um ponto de junção. As medidas dos sinais analíticos foram efetuadas utilizando um espectrofotômetro Femto, modelo 600 Plus, com saída de dados serial do tipo RS 232, equipado com uma cela de fluxo de vidro (ca. $180 \mu \mathrm{L}$ ) com caminho óptico de $10 \mathrm{~mm}$. Os valores de absorbância foram registrados no computador usando o software Hyperterminal da Microsoft, já disponível no sistema operacional Windows XP, ligado por um cabo DB9-DB9 de comunicação serial e os valores de máxima absorbância do sinal transiente determinado pelo software Excel da Microsoft.

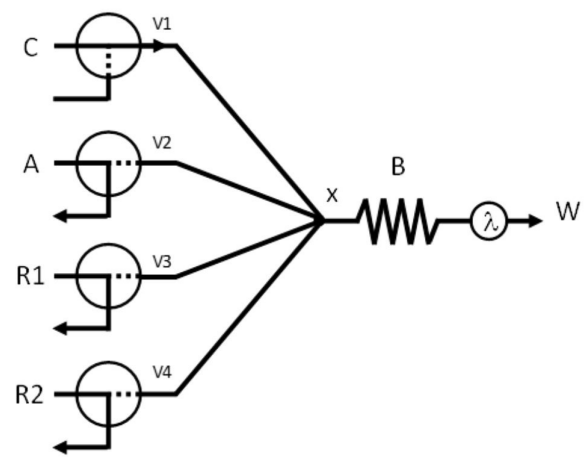

Figura 1. Diagrama de fluxo do módulo de análises. $C=$ transportador; $A$ = amostra; $R 1=$ solução de ácido ascórbico $0,5 \%(\mathrm{~m} / \mathrm{v}) ; R 2=$ solução de 1,10-fenantrolina $0,25 \%(\mathrm{~m} / \mathrm{v}) ; B=$ bobina de reação, $50 \mathrm{~cm}$ de comprimento, $0,8 \mathrm{~mm}$ de diâmetro interno; $l=$ espectrofotômetro $(512 \mathrm{~nm}) ; \mathrm{V} 1, \mathrm{~V} 2$, V3 e V4 = válvulas solenoide de 3 vias, $x=$ junção e $W=$ descarte

O programa de controle e acionamento das válvulas solenoide foi escrito com o software Arduino 1.04 e gravado no microcontrolador do Arduino. A análise química inicia com todas as válvulas desligadas e o fluido transportador passando continuamente pelo percurso analítico. A sequência e o tempo de acionamento das válvulas V1, V2, V3 e V4, são apresentados na Tabela 1. Alíquotas de amostra e reagentes são adicionadas no percurso analítico, formando a partir do ponto de junção $(\mathrm{x})$ em uma sequência ternária, que corresponde às alíquotas das soluções da amostra, do ácido ascórbico e da solução 1,10-fenantrolina. Esta zona de amostra foi conduzida pela solução transportadora através da bobina de reação (B) $50 \mathrm{~cm}$ até a cela de fluxo para medidas espectrofotométricas a $512 \mathrm{~nm}$.

O procedimento foi aplicado à análise de 5 amostras de águas coletadas no Rio Piracicaba e em lagoas artificiais na Escola superior de Agricultura Luiz de Queiroz, na cidade de Piracicaba. Para efeito de comparação, estas foram analisadas por espectrofotometria de absorção atômica com chama (FAAS).

\section{Construção da interface e programa de controle}

Para controle das válvulas solenoide foi utilizado um circuito integrado ULN2803 e fonte de alimentação comercial de corrente contínua de $12 \mathrm{~V}$, ligados conforme esquema da Figura 2. O circuito integrado e as ligações elétricas foram montados em uma placa de matriz de contato, não necessitando de soldas. O circuito esquemático e os componentes utilizados para a construção do Arduino podem ser acessados gratuitamente pelo site http://arduino.cc/en/uploads/Main/ Arduino_Uno_Rev3-schematic.pdf.

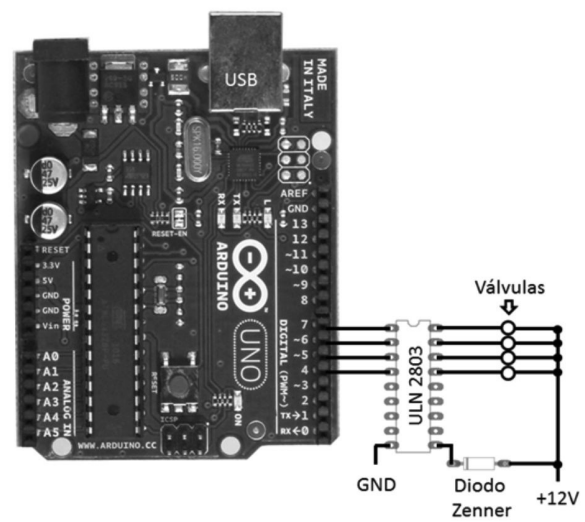

Figura 2. Esquema de ligação elétrica da placa do Arduino, circuito integrado ULN2803 e as válvulas solenoide

O software open source para programação do Arduino pode ser adquirido no site do desenvolvedor (www.arduino.cc), ${ }^{11}$ assim como exemplos e tutoriais de uso. Os comandos utilizados para a criação do programa de controle das válvulas solenoide são apresentados na Tabela 2. A função void setup realiza as ações dentro dos colchetes uma única vez, geralmente utilizada nas configurações iniciais. A função pinMode ativa as portas digitais (0 a 13), como saída (OUTPUT) ou entrada

Tabela 1. Sequência e tempos de acionamento das válvulas solenoide na determinação de ferro total em amostras de água. Os números 0 e 1 indicam a válvula desligada ou ligada, respectivamente

\begin{tabular}{|c|c|c|c|c|c|}
\hline Etapas & V1 & $\mathrm{V} 2$ & V3 & V4 & Tempo (s) \\
\hline Preenchimento do canal da amostra & 1 & 1 & 0 & 0 & 10 \\
\hline Limpeza do percurso analítico & 0 & 0 & 0 & 0 & 20 \\
\hline Adição da amostra* & 1 & 1 & 0 & 0 & 1 \\
\hline Adição da solução de ácido ascórbico* & 1 & 0 & 1 & 0 & 1 \\
\hline Adição da solução de 1,10-fenantrolina* & 1 & 0 & 0 & 1 & 1 \\
\hline Medida espectrofotométrica & 0 & 0 & 0 & 0 & 60 \\
\hline
\end{tabular}

*etapas repetidas em sequência por cinco vezes. 
de dados (INPUT). A função void loop realiza as ações dentro dos colchetes de forma cíclica, em um laço eterno. A função digitalWrite, indicada como $\mathrm{HIGH}$, liga a porta digital e LOW, desliga a porta digital e o delay estabelece o tempo de espera em milisegundos. Neste trabalho foram usadas somente quatro portas digitas (4 a 7), como apresentado na Figura 2. O código escrito foi gravado no microcontrolador que passa a realizar as ações de forma independente e contínua.

Tabela 2. Programa Arduino de acionamento das válvulas solenoide para o gerenciamento das soluções. Comentário da função de comando em itálico e precedido de "//"

Funções para controle das válvulas solenoide

void setup ( )

$\{$ pinMode $(4$, OUTPUT); pinMode (5, OUTPUT); pinMode (6, OUTPUT);

Ilativa a porta 4 (V1) como saída Ilativa a porta 5 (V2) como saída llativa a porta 6 (V3) como saída pinMode (7, OUTPUT); \} Ilativa a porta 7 (V4) como saída void loop ()

\{ digitalWrite(4, HIGH); I/abre a V1, desvia o fluxo do transportador digitalWrite(5, HIGH); I/abre a V2, preenche canal da amostra

delay(10000);

digitalWrite(4, LOW);

digitalWrite(5, LOW);

delay(20000);

for (count $=0$; count $<5$; count ++ ) //cria ciclo de amostragem ternária $\{$ digitalWrite $(4, \mathrm{HIGH})$;

digitalWrite(5, HIGH); I/adiciona amostra

delay(1000);

digitalWrite(5, LOW);

digitalWrite $(6, \mathrm{HIGH})$

delay(1000);

digitalWrite(6, LOW);

digitalWrite(7, HIGH);

delay(1000);

digitalWrite(7, LOW); \}

digitalWrite(4, LOW); I/conduz zona de amostra para medida

delay(60000); \}

\section{Aplicação do sistema}

As dimensões do módulo de análises do sistema de multicomutação e concentração dos reagentes do sistema proposto utilizaram as melhores condições obtidas por Feres e Reis ${ }^{12} \mathrm{e}$ os resultados obtidos são apresentados na Tabela 3. Estes resultados foram comparados aos apresentados por esses autores, mostrando similaridade das figuras de mérito, com melhor desempenho de consumo de reagentes e geração de resíduo. Este se deve a diferenças na configuração do sistema e ao número de repetições na etapa de amostragem binária, em que os autores utilizaram dez repetições e neste trabalho esta etapa foi reduzida para cinco. Podemos observar na Figura 3, que os sinais transientes das determinações analíticas são apropriados a um sistema de análises em fluxo com características similares a outros sistemas de análises em fluxo por multicomutação.,

Após a otimização dos tempos de acionamento das válvulas solenoide o programa gravado no microcontrolador permite que o sistema trabalhe de forma autônoma sem a necessidade do computador, utilizando a conexão com o cabo USB somente para a alimentação de $5 \mathrm{~V}$. Este pode ainda ser substituído por uma fonte alternativa como uma bateria. Este sistema pode ser facilmente adaptado a uma configuração portátil em determinações de campo.

Os resultados obtidos para determinação de ferro em cinco amostras de águas são mostrados na Tabela 4, juntamente com os resultados do procedimento de referência (FAAS). Os resultados dos procedimentos não apresentaram diferença significativa pelo teste de Tukey a $95 \%$ de confiança quando comparados.
Tabela 3. Características analíticas do sistema de análises em fluxo com multicomutação controlado pelo microcontolador Arduino e dados da literatura

\begin{tabular}{lcc}
\hline Parâmetro & Proposto & Literatura $^{12}$ \\
\hline Resposta linear $\left(\mathrm{mg} \mathrm{L}^{-1}\right)$ & $0-5$ & $0-10$ \\
Coeficiente de regressão $\left(\mathrm{R}^{2}\right)$ & 0,9994 & 0,9986 \\
Coeficiente de variação $(\%)$ & 2,9 & 1,0 \\
Limite de detecção $\left(\mathrm{mg} \mathrm{L}^{-1}\right)$ & 0,1 & 0,5 \\
Consumo de 1,10-fenantrolina $(\mu \mathrm{g})^{*}$ & 208 & 500 \\
Geração de resíduo $(\mathrm{mL})^{*}$ & 1,3 & 2,4 \\
Frequência de amostragem $\left(\mathrm{h}^{-1}\right)$ & 45 & 40 \\
\hline
\end{tabular}

* Por determinação.

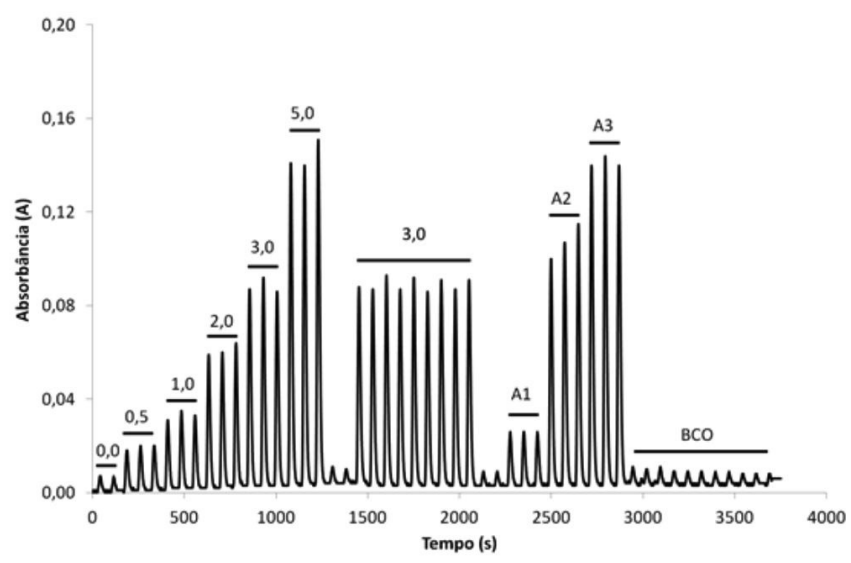

Figura 3. Sinais transientes das soluções de referência de 0 a $5 \mathrm{mg} \mathrm{L}^{-1}$ de ferro e amostras de água, obtidos pelo sistema de análise em fluxo controlado pela interface Arduino

Tabela 4. Concentrações de ferro determinadas pelo sistema de análise em fluxo controlado pelo arduino (FS-Arduino) e por espectrofotometria de absorção atômica (FAAS). A-D - amostras de lagoas artificiais; E-amostra do Rio Piracicaba

\begin{tabular}{ccc}
\hline \multirow{2}{*}{ Amostras } & FS-Arduino* & FAAS* \\
\cline { 2 - 3 } & & $\mathrm{mg} \mathrm{L}^{-1}$ \\
\hline A & $0,59 \pm 0,06$ & $0,63 \pm 0,01$ \\
B & $3,92 \pm 0,31$ & $3,81 \pm 0,01$ \\
C & $5,15 \pm 0,13$ & $4,49 \pm 0,06$ \\
D & $3,99 \pm 0,12$ & $3,81 \pm 0,01$ \\
E & $6,12 \pm 0,14$ & $5,82 \pm 0,05$ \\
\hline
\end{tabular}

*Médias e desvios de 3 replicatas.

\section{CONCLUSÕES}

O perfil dos sinais transientes, as figuras de mérito semelhante aos da literatura e os teores de ferro similares nos procedimento proposto e comparativo, fornecem indicativos que o gerenciamento das soluções realizado pelo Arduino é adequado e possui desempenho similar às interfaces precursoras.

Os componentes eletrônicos e a plataforma Arduino podem ser adquiridos a baixo custo no comércio, com valor aproximado de US\$ 35,00. Sua montagem é simples, não necessitando de mão-de-obra especializada. O desempenho da interface, avaliada pela determinação de ferro em água, atendeu plenamente as expectativas.

O sistema de controle das válvulas solenoide pode ser estendido a 
outros dispositivos em sistemas de análises em fluxo, como válvulas rotatórias, microbombas solenoide e bombas de seringa, alterando somente o software de controle.

\section{AGRADECIMENTOS}

Ao suporte financeiro FAPESP (proc. no 2011/23498-9 e 2012/08205-8), e bolsa do CNPq (proc. no 162593/2011-2).

\section{REFERÊNCIAS BIBLIOGRÁFICAS}

1. Reis, B. F.; Giné, M. F.; Zagatto, E. A. G.; Lima, J. L. F. C.; Lapa, L. A.; Anal. Chim. Acta 1994, 293, 129.

2. Růžička, J.; Hansen, E.H.; Mosbaek, H.; Anal. Chim. Acta 1977, 92, 235.

3. Lima, J. L. F. C.; Santos, J. L. M.; Dias, A. C. B.; Ribeiro, M. F. T.; Zagatto, E. A. G.; Talanta 2004, 64, 1091
4. Martelli, P. B.; Reis, B. F.; Kronka, E. A. M.; Bergamin Filho, H.; Korn, M.; Zagatto, E. A. G.; Lima, J. L. F. C.; Araujo, A. N.; Anal. Chim. Acta 1995, 308, 397.

5. Kronka, E. A. M.; Reis, B. F.; Vieira, J. A.; Blanco, T.; Gervasio, A. P. G.; Quim. Nova 1997, 20, 372.

6. Ruzicka, J.; Hansen, E. H.; Anal. Chim. Acta 1975, 78, 145.

7. Ródenas-Torralba, E.; Rocha, F. R. P.; Reis, B. F.; Morales-Rubio, A.; de La Guardia, M.; J. Autom. Methods Manag. Chem. 2006, 1.

8. Lima, R. S.; Santos, V. B.; Guerreiro, T. B.; Araújo, M. C. U.; Gaião, E. N.; Quim. Nova 2011, 34, 135.

9. Onacak, T.; Instrumentation Science and Technology 2006, 34, 347.

10. Fonseca, A.; Raimundo, I. M.; Rohwedder, J. J. R.; Lima, R. S.; Araújo, M. C. U.; Anal. Bioanal. Chem. 2010, 396, 715.

11. http://www.arduino.cc, acessada em Novembro 2012.

12. Feres, M. A.; Reis, B. F.; Talanta 2005, 68, 422. 